\title{
PREDICTORS OF ELEVATED TUMOUR NECROSIS FACTOR $\alpha$ LEVEL IN OBSTRUCTIVE SLEEP APNOEA SYNDROME
}

\author{
Silke Ryan, MD*, , Cormac T. Taylor, PhD* and Walter T. McNicholas, MD*,\# \\ *Sleep Research Laboratory, St. Vincent's University Hospital, Dublin, Ireland \\ \#The Conway Institute of Biomolecular and Biomedical Research, University College Dublin, Dublin, Ireland
}

WINNING ABSTRACT: Circulating levels of TNF- $\alpha$ are elevated in Obstructive Sleep Apnoea Syndrome (OSAS) and likely contribute to associated cardiovascular diseases. Furthermore, TNF- $\alpha$ has been suggested as a mediator of excessive daytime sleepiness (EDS). We investigated the predictors of TNF- $\alpha$ in OSAS in large, well-selected patient and control cohorts.

We undertook a prospective study of 30 non-OSAS (including 22 non-sleepy controls and 8 sleepy non-apnoeic snorers), 36 mild-moderate OSAS and 31 severe OSAS male subjects. All groups were closely matched in age, BMI, smoking status, blood pressure and lipid profile. All subjects were free of other disease and were not taking regular medication. Serum for TNF- $\alpha$ assay was drawn following polysomnography (PSG) in all subjects. 49 patients were commenced on CPAP therapy within one week following PSG; sleep studies and TNF- $\alpha$ measurements were repeated 6 weeks later.

TNF- $\alpha$ was higher in OSAS patients than controls $(p<0.001)$. In multivariate analysis, TNF- $\alpha$ was independently associated with the desaturation index $(r=0.399, p<0.001)$, the Epworth Sleepiness Score (ESS) $(r=0.243, p=0.005)$ and total cholesterol $(r=0.216, p=0.018)$. Furthermore, levels were higher in sleepy non-OSAS patients than in controls $\left(4.49[3.35,6.94] \mathrm{pg}^{-\mathrm{ml}^{-1}} \mathrm{vs}^{-}\right.$ 2.46[1.66,3.40]; $p=0.002)$ but lower than in severe OSAS patients $(6.32[5.70,8.17] ; p=0.03)$. CPAP significantly lowered TNF- $\alpha$ (from $5.56 \pm 2.10$ to $4.13 \pm 2.99 \mathrm{pg} \cdot \mathrm{ml}^{-1} ; \mathrm{p}=0.004$ ).

The severity of intermittent hypoxia is the strongest predictor of TNF- $\alpha$ level supporting a key role of inflammation in the cardiovascular pathophysiology of OSAS. Furthermore, TNF- $\alpha$ is independently associated with EDS.

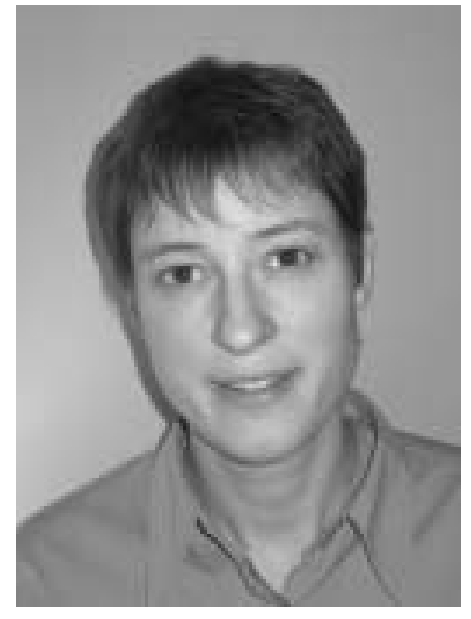

Silke Ryan

Sleep Research Laboratory, St. Vincent's University Hospital and The Conway Institute of Biomolecular and

Biomedical Research, University College Dublin, Dublin, Ireland

\section{MY JOB AND THE UNIT IN WHICH I WORK}

I am a Specialist Registrar in Respiratory Medicine and have spent the last 3 yrs in full-time research to complete a $\mathrm{PhD}$ thesis. The work, as presented in my abstract, has been the product of an ongoing intense and exciting collaboration between the Sleep Disorders Unit at St. Vincent's University Hospital (Dublin, Ireland), under the directorship of Prof. Walter McNicholas, Past President of the European Respiratory Society, and the hypoxia research group led by Prof. Cormac Taylor in the Conway Institute at University
College Dublin (Dublin). The Sleep Laboratory at St. Vincent's Hospital is the largest unit in the country dealing with sleeprelated breathing disorders. We mainly see patients suffering from obstructive sleep apnoea syndrome (OSAS) and we perform $\sim 250$ diagnostic sleep studies per year. In addition, we spend a great deal of time initiating and supervising treatment with continuous positive airway pressure (CPAP) therapy. Furthermore, the laboratory has great expertise in the management of breathing disorders related to various respiratory diseases, such as chronic obstructive pulmonary disease (COPD) and asthma. The Conway Institute at University College Dublin is an institution with state-of-the-art facilities targeted towards biomedical and biomolecular research. The research group led by Prof. Cormac Taylor has an international reputation in investigating cell and molecular mechanisms of hypoxia. The combination of the established clinical and physiological research strengths of the Sleep Research Laboratory and the extensive research record of Prof. Taylor's group in the cellular and molecular responses to hypoxia allows us to implement a programme of translational research. This research is directed towards a better understanding of the consequences for disorders associated with chronic intermittent hypoxia, such as OSAS.

\section{MY WINNING ABSTRACT AS PART OF MY RESEARCH}

My PhD, which I started in July 2003, is entitled "Activation of inflammatory pathways by intermittent hypoxia and its role in the cardiovascular pathophysiology of obstructive sleep apnoea syndrome". OSAS is a highly prevalent disorder affecting $\sim 4 \%$ of males and $2 \%$ of females [1], and with the growing incidence of obesity worldwide the prevalence of OSAS is also steadily growing. The predominant morbidity of OSAS is cardiovascular and OSAS is an independent risk factor for cardiovascular diseases, particularly systemic arterial 
hypertension but also coronary artery disease, congestive cardiac failure and cerebrovascular accidents [2-4]. The mechanisms by which OSAS predispose to cardiovascular disease remain unclear. The pathogenesis is most likely multifactorial and potential mechanisms include sympathetic excitation, inflammation, vascular endothelial dysfunction and metabolic dysregulation [5]. However, it is likely that for OSAS the typical cyclical pattern of intermittent hypoxia and reoxygenation plays a significant role in the pathophysiology. In particular, the intermittent re-oxygenation that distinguishes intermittent from sustained hypoxia resembles reperfusion injury and may result in the development of atherosclerosis. While the detailed molecular mechanisms underlying the cellular response to intermittent hypoxia remain unknown, the cellular responses to sustained hypoxia are well described and characterised by alteration in the expression of a number of adaptive genes, including stress-related genes and corresponding proteins that are necessary to maintain homeostasis [6]. Cells have developed protective transcriptional strategies in order to respond to sustained hypoxia. The master regulator in sensing hypoxic conditions and in integrating an adaptive response via gene expression is the transcription factor hypoxia-inducible factor-1 (HIF-1). HIF-1 activates transcription of genes encoding proteins that will either increase oxygen delivery (e.g. vascular endothelial growth factor, erythropoietin, endothelin-1) or achieve metabolic adaptations under conditions of reduced oxygen availability (e.g. glucose transporter-1 and glycolytic enzymes). Such factors mediate an adaptive response to hypoxia directed towards increasing tissue perfusion and oxygenation, and hence overcoming the initial hypoxic insult [7].

Sustained hypoxia also leads to the activation of other transcription factors, such as nuclear factor (NF)- $\kappa B$ [8]. NF$\kappa \mathrm{B}$ plays a central role in various physiological and pathophysiological processes. It is the key transcription factor in the inflammatory response and activated NF- $\mathrm{BB}$ controls the expression of many genes, including those encoding inflammatory cytokines, such as tumour necrosis factor (TNF)- $\alpha$ and interleukin (IL)-6, chemokines such as IL-8, adhesion molecules such as intercellular adhesion molecule- 1 and cell receptors [9]. These mediators have also been linked to the pathogenesis of atherosclerosis and hypertension [10]. A number of reports have shown an increase of circulating levels of these markers in OSAS and a significant fall with effective CPAP therapy [11-13].

We have developed a novel in vitro model of intermittent hypoxia and generated data indicating that intermittent hypoxia leads to a selective dose-dependent activation of pro-inflammatory events mediated by NF- $\kappa \mathrm{B}$ without significant activation of the protective HIF-1-dependent pathway [14]. These findings demonstrate that the intermittent hypoxia associated with OSAS may selectively activate molecular pathways that are pro-atherogenic; in this way the cellular response to intermittent hypoxia differs greatly from the response to sustained hypoxia.

At the 2006 European Respiratory Society Annual Congress, we presented data translating the findings of our cell culture model into the clinical picture of OSAS; in a large prospective study, we have demonstrated a significant relationship between NF- $\kappa$ B-dependent genes, TNF- $\alpha$ and IL- 8 , and sleeprelated oxygen desaturation occurring in OSAS patients [15]. Furthermore, we have demonstrated that CPAP therapy is an effective treatment for lowering this inflammatory response.

These results support our central hypothesis of a selective activation of the transcription factor NF- $\mathrm{BB}$ by OSAS leading to an inflammatory response that may contribute to the development of cardiovascular complications in this disorder. Furthermore, the data support a key role of TNF- $\alpha$ and IL-8 as cardiovascular risk markers in OSAS.

\section{THE IMPACT OF MY WORK ON CLINICAL OR RESEARCH PRACTICE}

In conclusion, the present data provide evidence for a specific role of intermittent hypoxia in inflammatory processes contributing to cardiovascular complications in OSAS. Further research is required to understand the detailed signalling mechanisms of NF- $\mathrm{BB}$ activation by OSAS and to explore the detailed molecular consequences of this event.

\section{REFERENCES}

1 Young T, Palta M, Dempsey J, Skatrud J, Weber S, Badr S. The occurrence of sleep-disordered breathing among middle-aged adults. N Engl J Med 1993; 328: 1230-1235.

2 Nieto FJ, Young TB, Lind BK, et al. Association of sleepdisordered breathing, sleep apnea, and hypertension in a large community-based study. Sleep Heart Health Study. JAMA 2000; 283: 1829-1836.

3 Shahar E, Whitney CW, Redline S, et al. Sleep-disordered breathing and cardiovascular disease: cross-sectional results of the Sleep Heart Health Study. Am J Respir Crit Care Med 2001; 163: 19-25.

4 Young T, Peppard P. Sleep-disordered breathing and cardiovascular disease: epidemiologic evidence for a relationship. Sleep 2000; 23: Suppl. 4, S122-S126.

5 McNicholas WT, Ryan S. Obstructive sleep apnoea syndrome: translating science to clinical practice. Respirology 2006; 11: 136-144.

6 Bunn HF, Poyton RO. Oxygen sensing and molecular adaptation to hypoxia. Physiol Rev 1996; 76: 839-885.

7 Semenza GL. $\mathrm{O}_{2}$-regulated gene expression: transcriptional control of cardiorespiratory physiology by HIF-1. J Appl Physiol 2004; 96: 1173-1177.

8 Cummins EP, Taylor CT. Hypoxia-responsive transcription factors. Pflugers Arch 2005; 450: 363-371.

9 Bonizzi G, Karin M. The two NF- $\kappa$ B activation pathways and their role in innate and adaptive immunity. Trends Immunol 2004; 25: 280-288.

10 Willerson JT, Ridker PM. Inflammation as a cardiovascular risk factor. Circulation 2004; 109, 21: Suppl. 1, II2-II10.

11 Yokoe T, Minoguchi K, Matsuo H, et al. Elevated levels of C-reactive protein and interleukin-6 in patients with obstructive sleep apnea syndrome are decreased by nasal continuous positive airway pressure. Circulation 2003; 107: 1129-1134.

12 Ohga E, Tomita T, Wada H, Yamamoto H, Nagase T, Ouchi Y. Effects of obstructive sleep apnea on circulating ICAM-1, IL-8, and MCP-1. J Appl Physiol 2003; 94: 179-184. 
13 Minoguchi K, Tazaki T, Yokoe T, et al. Elevated production of tumor necrosis factor- $\alpha$ by monocytes in patients with obstructive sleep apnea syndrome. Chest 2004; 126: 1473-1479.

14 Ryan S, Taylor CT, McNicholas WT. Selective activation of inflammatory pathways by intermittent hypoxia in obstructive sleep apnea syndrome. Circulation 2005; 112: 2660-2667.

15 Ryan S, Taylor CT, McNicholas WT. Predictors of elevated nuclear factor $\kappa \mathrm{B}$-dependent genes in obstructive sleep apnea syndrome. Am J Respir Crit Care Med 2006; 174: 824-830. 